\title{
NEOTECTÓNICA Y GEOFÍSICA DE LA FALLA AGUACALIENTE EN LOS VALLES CORIS Y EL GUARCO, COSTA RICA
}

\author{
Walter Montero ${ }^{* 1} \&$ Sarah Kruse ${ }^{2}$ \\ ${ }^{1}$ Escuela Centroamericana de Geología, Universidad de Costa Rica \\ ${ }^{2}$ Department of Geology, University of South Florida \\ *Autor para contacto: wmontero@geologia.ucr.ac.cr
}

(Recibido: 25/09/06 ; aceptado: 25/01/07)

\begin{abstract}
In the Coris and Guarco valleys, we conducted geomorphic and geophysical studies and compiled geological, hydrogeological and trench information in order to define the traces of the Aguacaliente fault. The principal active trace of this fault trends west-northwest and is located in the southern sector of the Coris and El Guarco valleys. Rivers and creeks bending between 50 and $450 \mathrm{~m}$ suggest it is a sinistral fault. The location of the fault trace is well constrained in certain parts of both valleys; elsewhere the fault trace is inferred using geomorphic criteria. The fault trace shows a wide deformation zone that covers between tens of meters to more than hundred meters. The lack of fault prominence and the wide fault zone are explained by the combination of a horizontal displacement fault traversing a topographically flat region with a Quaternary sedimentary fill, poorly consolidated in its upper portion, and erosion and sedimentation rates that are greater than the rate of seismic activity along the fault.

A second west-northwest fault trace is defined along the northern border of the Coris valley on the boundary of the Carpintera hills. To the east, this fault trace appears to end in a series of short northeast-trending faults. Along with geomorphic data and an interpretation of magnetic data, the fault traces suggest a normal component of displacement. These traces could be inactive.

Key words: Morphotectonics, neotectonics, electrical method, magnetic method, hydrogeology, Aguacaliente Fault.

RESUMEN: En los valles de Coris y de El Guarco se realizaron estudios geomórficos y geofísicos y se recopiló información geológica e hidrogeológica, así como de trincheras para definir las trazas de la falla Aguacaliente. La traza principal activa de esta falla tiene un rumbo oeste-noroeste y se localiza sobre el sector sur de los valles de Coris y de El Guarco. Desvíos entre 50 y 450 metros en ríos y quebradas, indican que es una falla de rumbo sinestral. La ubicación de esta traza de falla está bien restringida en sectores limitados en ambos valles, mientras que en la mayor parte se ha inferido usando criterios principalmente geomórficos. Asimismo, la traza de falla muestra una zona ancha de deformación, que puede tener entre unas decenas de metros hasta más de cien metros. La poca prominencia de la falla y el ancho de la zona de falla se explican por la combinación de varios factores, como son una falla de desplazamiento horizontal atravesando un relleno sedimentario cuaternario de topografía bastante plana. Este relleno se encuentra poco consolidado y las tasas de erosión y sedimentación superan la tasa de actividad sísmica de la falla.

Una segunda traza de falla con un rumbo oeste-noroeste se interpreta en el lado norte del valle de Coris, en el límite con los cerros de la Carpintera. Esta traza parece terminar al este en una serie de fallas cortas de rumbo noreste. De acuerdo
\end{abstract}

MONTERO, W. \& KRUSE, S., 2006: Estudio neotectónico y geofísico de la falla Aguacaliente en los valles de Coris y de El Guarco.- Rev. Geol. Amér. Central, 34-35: 43-58. 
con la expresión geomórfica y una interpretación de los datos magnéticos, el anterior conjunto de trazas sugieren tener una componente normal y podrían estar inactivas.

Palabras clave: Morfotectónica, neotectónica, método eléctrico, método magnético, hidrogeología, Falla Aguacaliente.

\section{INTRODUCCIÓN}

El Cinturón Deformado del Centro de Costa Rica (CDCCR) constituye un sistema ancho de fallas y pliegues activos, el cual define el límite oeste entre la microplaca Panamá y la placa Caribe (ver recuadro en Fig. 1a). Entre las fallas activas del CDCCR se encuentra la falla Aguacaliente (Fig. 1a), con la cual se asocian diversos terremotos históricos de magnitudes intermedias (5,0 $<\mathrm{M}_{\mathrm{s}}<6,5$ ) (Montero et al., en prensa). Entre las secuencias sísmicas más dañinas asociadas con esta falla se encuentra la ocurrida entre 1910 y 1912 , en la que destaca el desastroso terremoto del 4 de mayo $\left(\mathrm{M}_{\mathrm{s}} 6,1\right)$, que destruyó la ciudad de Cartago y otros poblados cercanos y el cual se ubicó unos kilómetros al sur de la anterior ciudad (Montero \& Miyamura,1981).

La falla Aguacaliente ha sido motivo de un estudio detallado al sur y suroeste de San José (Montero et al., en prensa). Con respecto al sector de la falla al sur de Cartago, se han realizado varios estudios (p.e. Montero et al., 1991; Woodward \& Clyde, 1993; Fernández \& Montero, 2002). En esta investigación se amplían los estudios anteriores, con investigaciones geomorfológicas y geofísicas y reuniendo la información geológica e hidrogeológica disponible, con el objetivo de definir mejor las características estructurales y geométricas de las trazas de la falla Aguacaliente en los valles de Coris y de El Guarco y, de esta manera, contribuir al conocimiento de la amenazas sísmica y por ruptura de falla asociadas con ella.

\section{METODOLOGÍA}

En este estudio se aplican técnicas morfotectónicas y geofísicas complementadas con datos geológicos e hidrogeológicos. Los criterios de geomorfología tectónica usados en este estudio son los usualmente aplicados en el reconocimiento de fallas activas, mediante imágenes de sensores remotos y observaciones de campo (p.e. Yeats et al., 1997).
Con respecto al estudio geofísico, este consistió primero, en la recolección de datos de resistividad y magnéticos en sectores donde se sospechaba la presencia de la falla. Los datos de resistividad fueron obtenidos a lo largo de perfiles usando un equipo de resistividad Campus Geopulse, el cual se usó en un dispositivo Wenner transversal de 48 electrodos. Los datos crudos obtenidos en el campo fueron luego invertidos para obtener las resistividades del terreno usando el paquete computacional Res2dinv (Loke \& Barker, 1996). Los datos magnéticos fueron recolectados usando un magnetómetro marca G-856 de la casa Geometrics, Inc., el cual tiene un sensor a 1,9 $\mathrm{m}$ arriba del nivel del terreno. Los datos fueron corregidos por las variaciones diurnas realizando un muestreo repetitivo en una estación base. Posteriormente se obtuvieron las líneas magnéticas, a partir de los datos. Estos fueron interpolados entre las líneas usando la opción de la interpolación cúbica con datos de redes del programa Matlab de Mathworks Inc.

Finalmente, para mejorar el conocimiento de la geología bajo el valle de Coris y elaborar modelos magnéticos acordes con ella, se obtuvo en el Servicio Nacional de Aguas Subterráneas, Riego y Avenamiento (SENARA), la información geológica resumida de los diversos pozos. Asimismo, el estudio hidrogeológico e hidroquímico realizado por Pérez (2000) en el valle de Coris, permitió obtener información sobre el flujo del agua subterránea en esta zona, el cual puede tener un control tectónico, lo cual se discute en el apartado correspondiente.

\section{LA FALLA AGUACALIENTE}

La falla Aguacaliente ha sido relacionada con actividad sísmica desde el trabajo de Dóndoli \& Torres (1954). Geomorfológicamente, la falla Aguacaliente se ha caracterizado, como veremos, por el alineamiento de promontorios truncados, valles lineales, ríos y quebradas desplazadas y adaptadas, alineamiento de fuentes termales 
y mineralizaciones sulfurosas (en los valles de Coris y de El Guarco), escarpes y sillas de falla (Woodward \& Clyde, 1993; Fernández \& Montero, 2002; Montero et al., en prensa). La falla se considera de desplazamiento predominante sinestral (Fernández \& Montero, 2002; Montero et al., en prensa).

La traza de la falla Aguacaliente tiene en general, una forma sinuosa con un rumbo variable entre ENE y WNW. Al sur de San José, el trazo principal de la falla pasa del lado sur de la loma Salitral (LS, Fig. 1a) y continúa hacia el este con un rumbo cercano al E-W hasta salir al valle de Coris (Fig. 1b). Más al este, la falla se ubica del lado sur de la ciudad de Cartago.

Diferentes trazas han sido interpretadas para la falla por diversos autores en los valles de Coris y de El Guarco (Dóndoli \& Torres,1954; Montero et al., 1991; Woodward \& Clyde, 1993; Montero, 1994; Fernández \& Montero, 2002). Lo anterior se debe a que la falla tiene una expresión geomorfológica débil, a la alta complejidad estructural y a los escasos afloramientos existentes, lo cual dificulta el cartografiado geológico. Es interesante observar que Woodward \& Clyde (1993) sugieren varias trazas de falla entre la loma Salitral y el sur de Cartago. Una de las trazas se ubica del lado norte del valle de Coris y termina al este en una serie de fallas cortas de un rumbo noreste, que limitan el lado este de los cerros de La Carpintera (CC, Fig. 1a). Una segunda traza se ubica del lado sur del valle de Coris y continúa al sur de la ciudad de Cartago. Woodward \& Clyde (1993) incluyen estas trazas dentro del sistema de falla Aguacaliente. A continuación se describen los estudios realizados.

\section{Investigaciones de la falla Agua Caliente en el Valle de Coris}

\section{Observaciones geomórficas}

El primer aspecto geomórfico que destaca es que el valle de Coris es una planicie localizada entre montañas por los lados norte, sur y oeste y abierto por el lado este (Fig. 1). Esto sugiere un posible control tectónico en el origen de este valle.
De acuerdo con entrevistas realizadas a personas mayores oriundas de la zona o con recuerdos de sus ancestros, el valle era sumamente pantanoso y por lo tanto mal drenado. Aún hoy se observan algunas zonas mal drenadas. Dóndoli \& Torres (1954) mencionan que los suelos son arcillosos y que se puede incluso diferenciar una fase arcillosa pantanosa (en la parte central-este), en la cual el agua afloraba todo el año. Lo anterior ha motivado que el sistema de drenaje en el valle de Coris haya sido muy alterado por el ser humano. Los cauces originales de varias de las quebradas han sido convertidos en canales de trazo rectilíneo. Esto se observa actualmente en los diversos sectores del valle. Esto se puede observar al comparar las ediciones 1:50 000 de la hoja Istarú del Instituto Geográfico Nacional (IGN) de los años 1961 y 1981. Asimismo, con respecto a la hoja 1: 10000 de la Hoja Tobosi del año 1991 (comparar Figs. 1b y 2). Uno de los ríos cuyo curso original ha sido alterado es el del río Coris. En la edición de la hoja Istarú de 1961 se muestra el curso original de ese río, el cual sugiere un posible desvío izquierdo, que se puede estimar en unos 700 metros (Fig. 1b, indicado entre las flechas con el número 1$)$.

Un aspecto geomórfico que sugiere la existencia de una falla en el lado sur del valle de Coris es la presencia de varios espolones facetados (EF, Fig. 2a) alineados. Otra evidencia de la falla, es que del lado norte de los espolones truncados anteriores, se encuentran las fuentes termales de Bermejo, las cuales se ubican en dos grupos de seis manantiales cada uno (Fig. 2a). El del lado occidental está alineado con un rumbo NE. El segundo, ubicado al oriente del anterior, incluye varias fuentes agrupadas y otra fuente termal ubicada al SW. Si se estima que este último grupo también tiene una disposición alineada al NE, los grupos alineados de las fuentes termales sugieren que el ascenso del agua caliente ocurre a lo largo de dos fracturas, que se interpretan como de tipo tensional. Estas pudieron formarse por una cupla sinestral de rumbo aproximado E-W, similar al rumbo propuesto para la falla (Fig. 2b). En la figura 2 a se muestra una traza de falla pasando por las fuentes termales y del lado norte de los espolones facetados. 

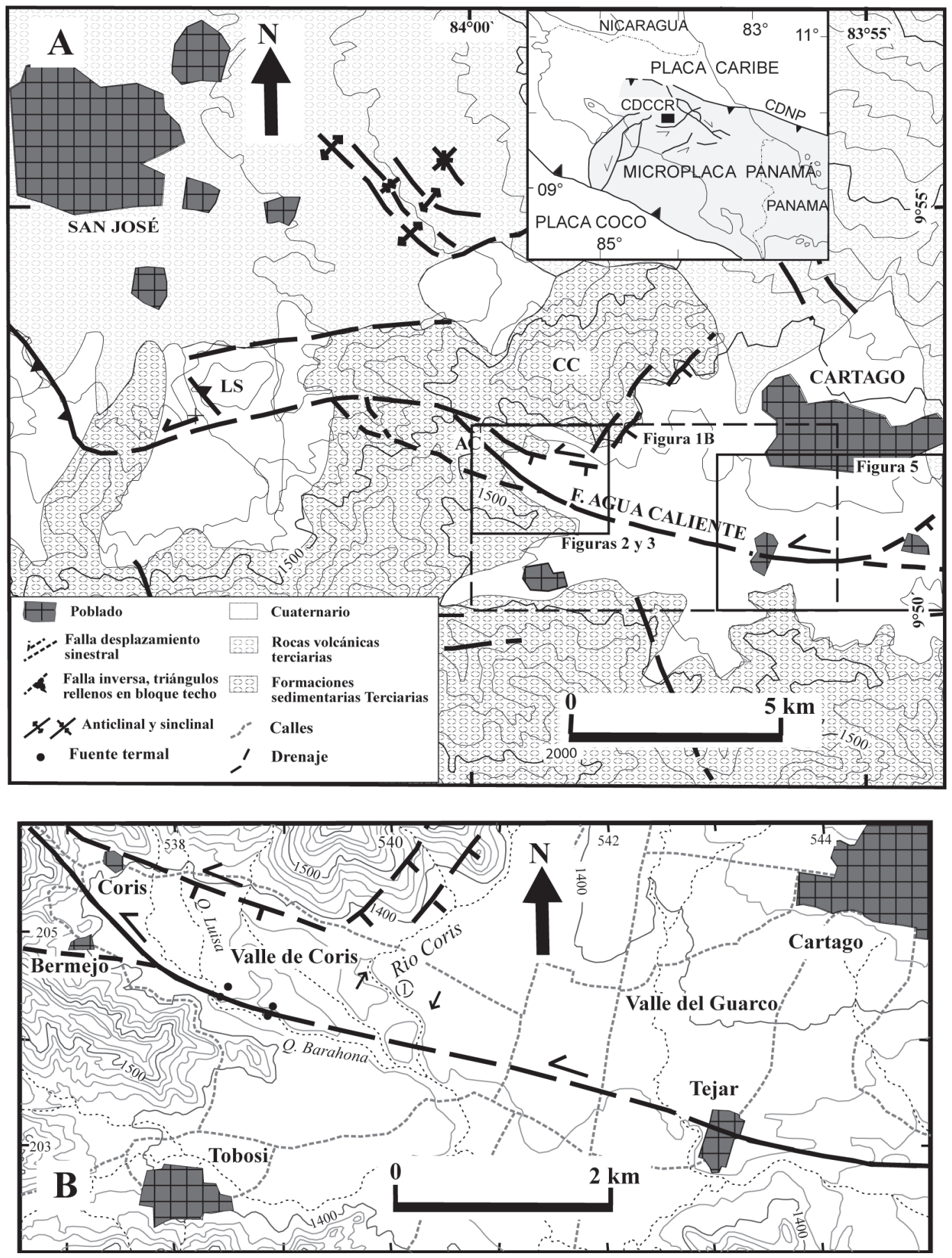

Fig. 1A: Falla Aguacaliente al sur de San José y de Cartago. Geología de Denyer \& Montero (1988). LS, AC y CC son respectivamente la loma Salitral, Alto Coris y los cerros La Carpintera. Los rectángulos muestran la ubicación de las figuras 1B, 2, 3 y 5 . En el recuadro se muestra el marco tectónico regional. CDCCR es el Cinturón Deformado del Centro de Costa Rica y CDNP es el Cinturón Deformado del Norte de Panamá.

Fig. 1B: Trazas de las fallas Aguacaliente en los valles de Coris y de El Guarco. Las flechas numeradas 1 muestran el desvío del río Coris. 


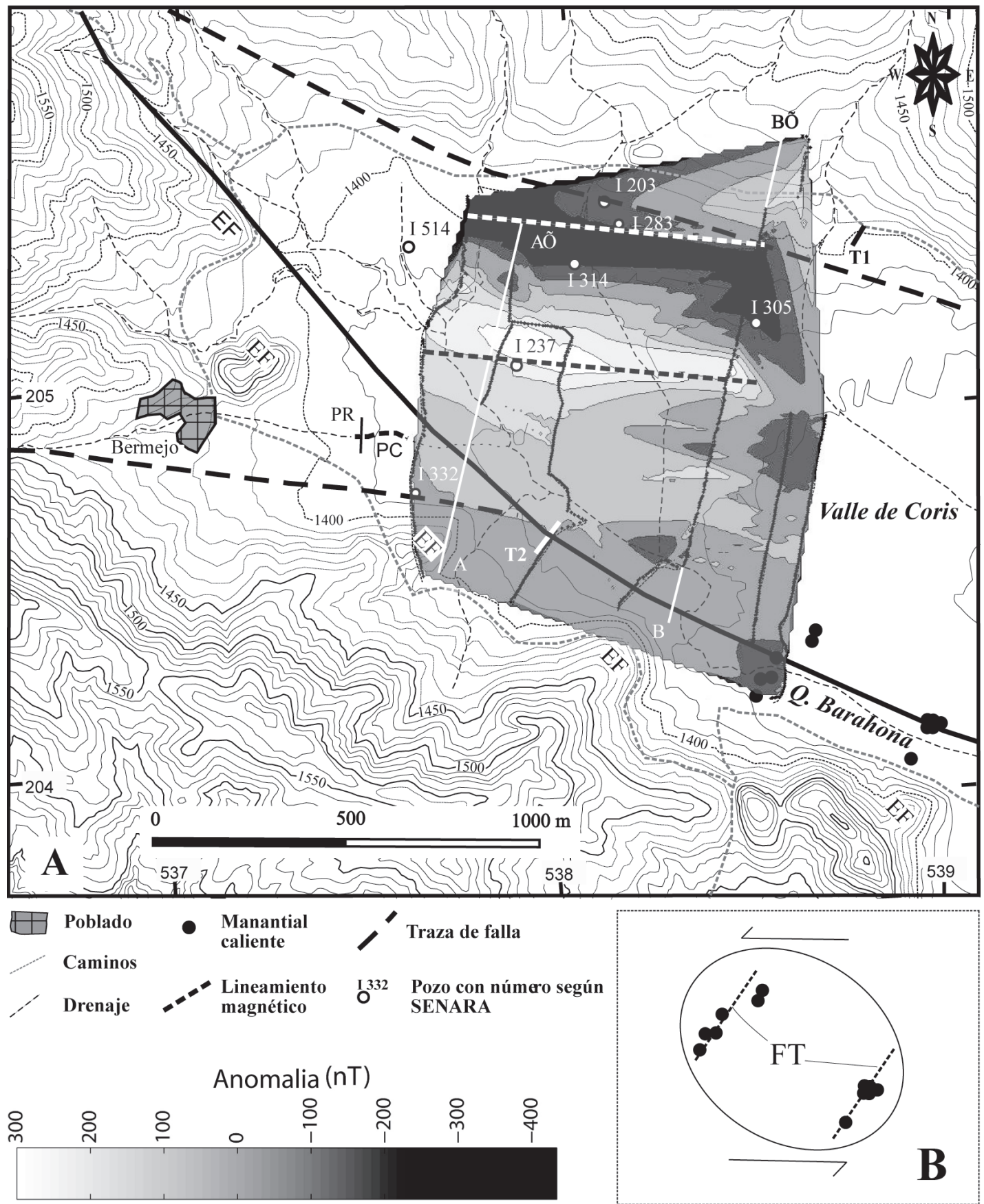

Fig. 2: Mapa magnético y topográfico del valle de Coris. Curvas de nivel provienen de las hojas Carpintera y Tobosi (1:10 000) del IGN con un intervalo cada $5 \mathrm{~m}$ por debajo de los $1420 \mathrm{~m}$ y cada $10 \mathrm{~m}$ sobre los $1420 \mathrm{~m}$. EF son los espolones facetados. PR y la línea negra delgada N-S muestra el perfil de resistividad eléctrica realizado transversal al paleocauce (PC), en trazos cortos. Se muestra la ubicación de la trincheras T1 y T2. Las líneas negras continuas transversales al valle muestran los perfiles magnéticos. Las líneas delgadas blancas AA’ y BB' muestran la ubicación de los perfiles magnéticos de la figura 4. B: Interpretación del origen de las fuentes termales de Bermejo, a lo largo de fracturas de tensión (FT) y asociadas a una cupla sinestral. 
Un aspecto geomórfico que se investigó en el sector suroeste del valle, fue una suave grada topográfica (1 m o menos), que baja del lado norte, la cual se reconoció por unos 200 m (Fig. 2a, línea segmentada indicada por PC). Esta morfología sugería una escarpita de falla o un elemento erosivo relacionado con algún contraste litológico. En un corte geológico transversal a la grada, encontrado en una quebrada, se determinó un contacto fuertemente inclinado entre depósitos aluviales gruesos (lado sur) y depósitos arcillo-limosos (lado norte). Para aclarar el origen de la anterior morfología se realizó un perfil eléctrico transversal a la grada. Los resultados de este estudio se verán posteriormente.

Una segunda traza de falla es sugerida al pie del frente de montaña del lado sur de los cerros de La Carpintera, el cual es bastante lineal, se muestra facetado al sur y tiene un rumbo oeste-suroeste. Del lado este de los cerros de La Carpintera se pueden observar varios lineamientos cortos de rumbo NE que pasan por sillas de falla, valles lineales y con facetamientos triangulares. El anterior conjunto de trazas se muestra en la figura 1.

\section{Geología e hidrogeología}

En el sector sur y oeste de los cerros que limitan el valle de Coris afloran las formaciones sedimentarias Peña Negra (Mioceno Medio), San Miguel (Mioceno Inferior a Medio) y Coris (Mioceno Medio) (Fig. 3). Las rocas volcánicas de la Formación La Cruz (Mioceno Superior cuspidal) se localizan en los cerros de La Carpintera, del lado norte del valle. Una descripción amplia de las anteriores formaciones se encuentra en Denyer \& Arias (1991). Por otro lado, sedimentos cuaternarios rellenan el valle de Coris, y dentro de estos se incluyen depósitos de pantano, lacustres, aluviales, lahares y coluvios.

Una traza de falla de rumbo E-W, que pasa ligeramente al sur de Bermejo, se puede interpretar de la geología, la cual pondría en contacto la Formación Peña Negra con la Formación Coris. Evidencias geomórficas de esta traza se encuentran al oeste y fuera de la zona de estudio.

Pérez (2000) analiza la hidrogeología del valle de Coris y obtiene las curvas isofreáticas y las direcciones de flujo del agua subterránea (Fig. 3). Aunque los datos fueron obtenidos con pocos pozos de control, un aspecto que sobresale de estas isofreáticas es que el flujo del agua subterránea ocurre a lo largo de una dirección WNW en la zona media norte del valle de Coris, la cual se encuentra limitada por zonas con fuertes gradientes en las isofreáticas en el lado central y norte del valle.

\section{Investigaciones en trincheras}

Woodward \& Clyde (1993) reportan la excavación de dos trincheras exploratorias a lo largo de dos posibles trazas de la falla Aguacaliente en el valle de Coris (T1 y T2, Fig. 2a). La trinchera T1 se realizó transversal a la traza de la posible falla ubicada en el lado norte del valle de Coris. En esta trinchera no se encontraron evidencias de deformación neotectónica, aunque las características geológicas y logísticas encontradas durante la excavación de la trinchera no fueron las apropiadas.

La trinchera T2 se ubicó en la llanura fluviolacustre, entre dos salientes truncados. En esta trinchera se encontró una zona de falla de unos $40 \mathrm{~m}$ de ancho, con una componente de desplazamiento de rumbo. Una datación realizada en una muestra de carbón recolectada en un horizonte desplazado proporcionó una edad de carbono 14, de $3665 \pm$ 145 años A.P. (Woodward \& Clyde, 1993).

\section{Investigaciones geofisicas}

Un levantamiento de resistividad con 48 electrodos en un dispositivo Wenner transversal, con un espaciamiento de $4 \mathrm{~m}$ entre electrodos, fue realizado en dirección N-S, transversal a la grada topográfica mencionada previamente (Fig. 2a). Los datos invertidos de la resistividad del terreno dieron una resolución hasta una profundidad de $20 \mathrm{~m}$. Sin embargo, no se encontró ninguna variación significativa a través de la grada topográfica La interpretación es que la grada topográfica ha resultado de la erosión laminar de los depósitos más finos ubicados del lado norte y que los depósitos observados en el corte de la quebrada corresponden con un banco remanente de un paleocanal. La grada topográfica asociada con 
el paleocanal se puede seguir hasta que se llega a un drenaje abandonado, el cual se unía con la quebrada Barahona (Fig. 2a).

Como parte de las investigaciones geofísicas realizadas, se tomaron datos magnéticos aproximadamente cada $6 \mathrm{~m}$, a lo largo de perfiles NNESSW, que cortaron el ancho del valle de Coris en su sector central. Los contornos de las anomalías se muestran en la figura 2a. El principal rasgo encontrado es un par de anomalías positivas y negativas (con una amplitud total de $600 \mathrm{nT}$ ), de un rumbo WNW-ESE, que son aproximadamente paralelas al borde norte del valle de Coris.

La figura 4 muestra modelos magnéticos sencillos para los perfiles largos que se ubican más al oeste y al este en la figura 2a. Estos modelos fueron elaborados, a propósito, conceptualmente muy sencillos y son inherentemente no únicos. La tendencia lineal de mejor ajuste y un valor constante fueron removidos de los datos crudos antes de dibujar cada uno de los perfiles. Los datos fueron proyectados de las tres líneas más cercanas en el perfil A-A'. En este perfil, los datos magnéticos se ajustan mejor con modelos que asumen una zona magnetizada ubicada entre $\sim 5$ y $\sim 200 \mathrm{~m}$ de profundidad, que se extiende entre las anomalías magnéticas alta y baja. El perfil ubicado más al este (B-B', Fig. 4) requiere de una zona magnetizada que corresponde con las rocas volcánicas de la Formación La Cruz, que afloran en el borde norte del valle. Los modelos asumen susceptibilidades razonables para las rocas volcánicas

Obsérvese que el flujo del agua subterránea tiende a ser paralelo al rumbo de la capa magnética y muy posiblemente fluya dentro de una capa lávica o de sedimentos ricos en componentes volcánicos. Sin embargo, las curvas isofreáticas y las líneas de flujo del agua subterránea fueron realizadas con pocos pozos de control.

\section{Investigaciones al sur del valle de El Guarco}

\section{Observaciones geomórficas}

Al sur del valle de El Guarco, la traza de la falla Aguacaliente puede ser determinada con base en las siguientes evidencias geomórficas (Fig. 5):
1) Un cambio de rumbo en el río Reventado al $\mathrm{S} 60^{\circ} \mathrm{E}$ al oeste de Tejar, con un posible desvío izquierdo de unos $450 \mathrm{~m}$ (Fig. 5, intervalo entre flechas numerado 2). Obsérvese que el trayecto del río es prácticamente $\mathrm{N}-\mathrm{S}$ aguas arriba y aguas abajo del posible desvío, antes de unirse al río Aguacaliente. 2) Entre los poblados de Tejar y de Hervidero, al sur del río Agua Caliente, se encuentran dos espolones montañosos facetados al $\mathrm{N}$ (EF, Fig. 5). Estos espolones facetados se alinean con los que se encuentran al sur del valle de Coris descritos previamente. Asimismo, al lado norte del espolón facetado de Hervidero, a lo largo del río Aguacaliente se ubica un manantial caliente (Fig. 5, círculo negro). Ambos espolones marcan el límite al norte de los afloramientos de las rocas sedimentarias terciarias, las cuales buzan aproximadamente hacia el sur. 3) Al este de Tejar, se encuentra una loma de rumbo $\mathrm{S} 70^{\circ} \mathrm{E}$ (Fig. 5, L1). Esta es seguida al E por otras dos lomas de rumbo similar (L2 y L3, Figs. 5 y 6). La parte sur de las lomas tienen un escarpe facetado al sur, de una altura máxima entre 15 y 20 m (Fig. 6b). 4) Aguas arriba de las lomas anteriores, la quebrada Cangrejal, que fluye al sur, cambia su curso al oriente, y luego al cortar entre las lomas L2 y L3 origina un valle encajado. Al salir de las lomas al sur, la quebrada tiene un desvío de $50 \mathrm{~m}$ a la izquierda (hacia el E; flecha numerada 3, Fig. 6a), antes de retomar un curso al sur hasta unirse al río Aguacaliente. Un desvío similar de unos $250 \mathrm{~m}$ se observa en la quebrada Molino, que se ubica al ENE de la anterior (flechas numeradas 4, Figs. 5 y 6a). Se puede interpretar que los desvíos aguas arriba anteriores se relacionan con un levantamiento tectónico de las lomas, provocando el suavizado del perfil del río aguas arriba y por lo tanto, el valle encajado entre las lomas L2 y L3 fue formado por un río antecedente.

\section{Investigaciones en trincheras}

Al suroeste y este de Tejar, Montero et al. (1991) realizaron varias trincheras. Tres trincheras se ubicaron al suroeste de Tejar, al sur de donde el río Reventado tiene un posible desvío al E (trincheras T3, T4 y T5, Fig. 5). En estas trincheras se encontró una secuencia de aluviones de diferentes granulometrías y paleosuelos, 


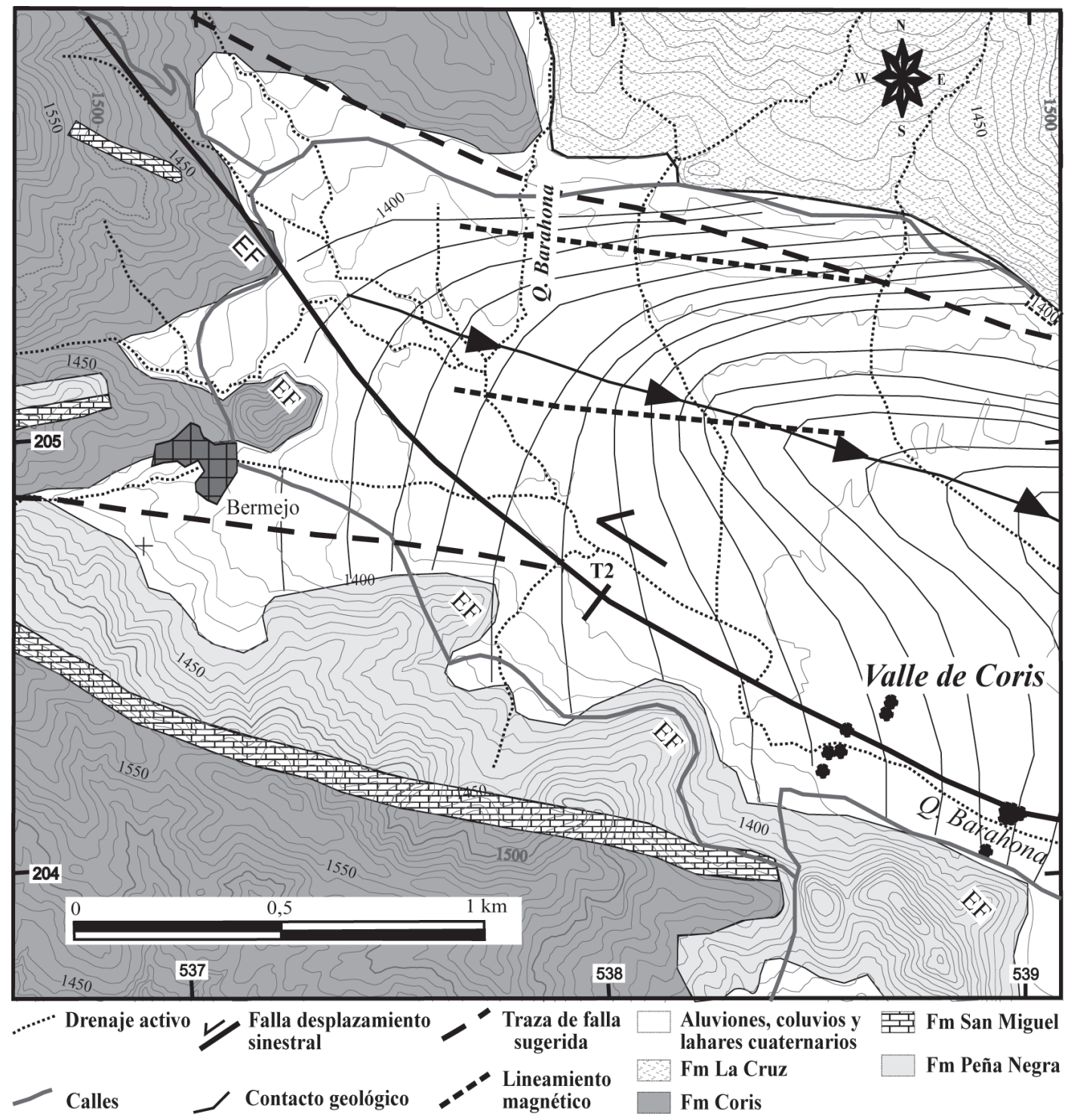

Fig. 3: Mapa geológico e hidrogeología del valle de Coris. Geología de Morales (1975) y terminología estratigráfica de Denyer \& Arias (1991). Las isofreáticas (líneas negras finas) y las líneas de flujo (línea negra con triángulos) son de Pérez (2000). Otras simbologías se muestran en la figura 2.

sin evidencia de deformación reciente. Al este de Tejar, se realizaron las trincheras T6, T7 y T8 (Fig. 6). Las trincheras T6 y T8 cortaron a través del escarpe de la loma L2. En esas trincheras, se encontraron depósitos laháricos del Pleistoceno Superior - Holoceno cortados por fallas, mientras que en la trinchera T7 se encontró fracturación tectónica (Montero et al., 1991). Estos autores interpretaron que la loma se originó por una transpresión local de la falla.

\section{Investigación geofísica}

Al este de Tejar, fue realizado un perfil de resistividad N-S de $250 \mathrm{~m}$ de largo, con 

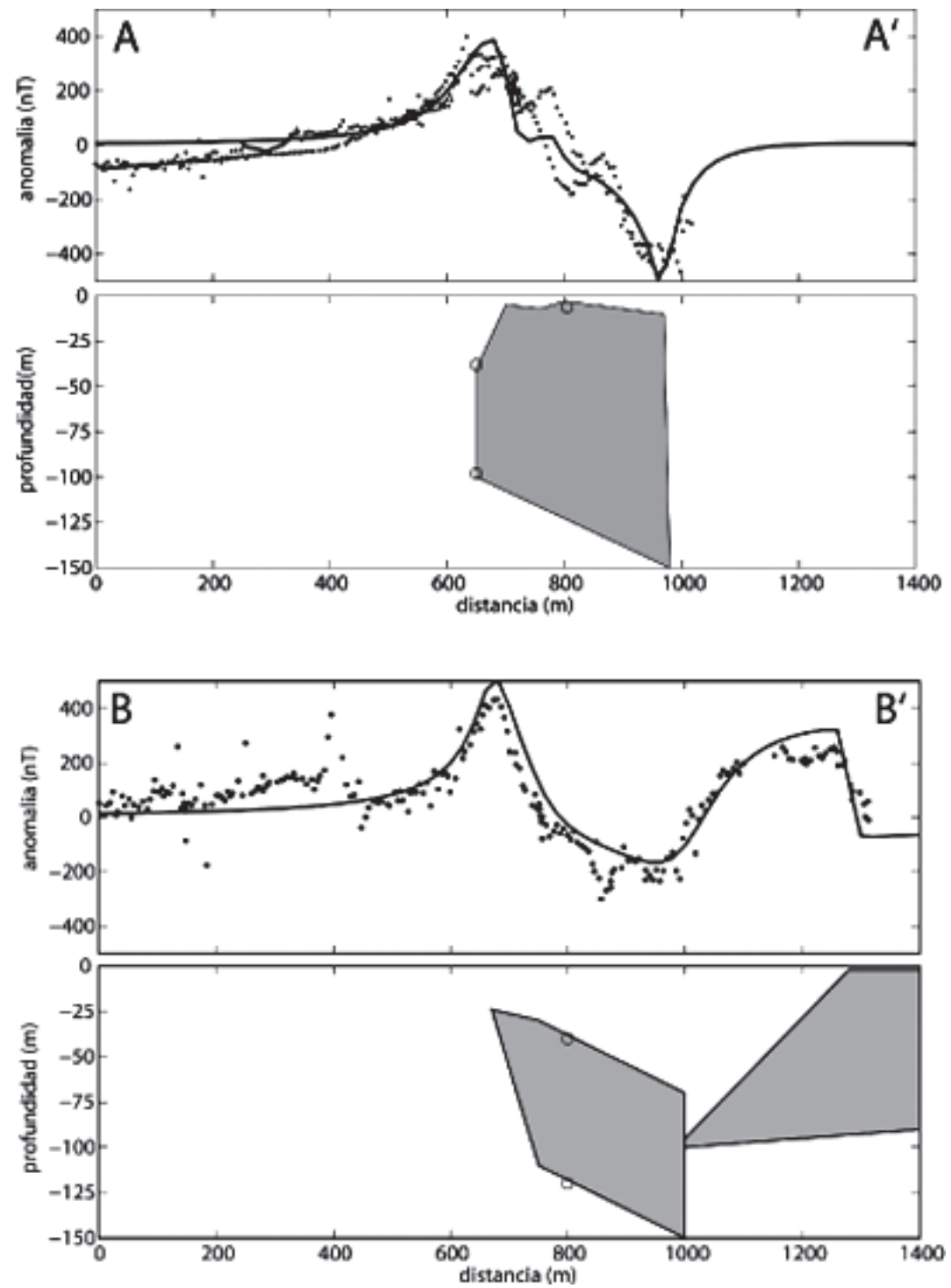

Fig. 4: Perfiles magnéticos del valle de Coris. Datos magnéticos y modelos mostrados para los perfiles A-A' y B-B' (Fig. 2). Los datos se muestran con puntos negros; las anomalías magnéticas correspondientes con los modelos son las líneas continuas. El bloque gris en el modelo correspondiente al perfil A-A' representa una zona con susceptibilidad de 0,04 (unidades MKS) con una longitud de $1 \mathrm{~km}$ a lo largo del rumbo. Los círculos representan límites de unidades descritas ya sea como lahares o como arenas de origen volcánico, extrapoladas de pozos cercanos. Los círculos ubicados a $650 \mathrm{~m}$ en A-A' provienen del pozo I-237, el círculo a $800 \mathrm{~m}$ proviene del pozo I-514. Los bloques grises en el modelo mostrado en B-B' representan bloques con una susceptibilidad de 0,08 y una longitud a lo largo del rumbo de $10 \mathrm{~km}$. Estos modelos son no-únicos.

un espaciamiento de $6 \mathrm{~m}$ entre electrodos, que cortó a través de la loma L2, en un sitio donde el escarpe tiene una altura de $\sim 10 \mathrm{~m}$ (Fig. 6). El resultado de la inversión de las lecturas de resistividad se muestra en la figura 7. La inversión tiene un error de un $7 \%$ en la raíz media cuadrática
(RMS), entre las observaciones y el modelo de resistividades. A ambos lados del escarpe de falla, las resistividades del terreno son altas en los $10 \mathrm{~m}$ superiores, con resistividades menores extendiéndose hasta $30 \mathrm{~m}$ o más por debajo de la superficie del terreno. El patrón de resistividades es similar 
al observado por Montero et al. (1991) a lo largo de un sondeo unidimensional realizado a través del valle al sur del escarpe. Ellos reconocen la capa superior de alta resistividad como lahares (verificado en trincheras), e infieren que la capa inferior de baja resistividad corresponde con sedimentos o paleolahares. La baja resistividad de la capa inferior sugiere que esta capa es más rica en arcilla que los lahares superiores.

El patrón estratigráfico de dos capas está claramente perturbado a lo largo de una zona aproximadamente vertical de unos $20 \mathrm{~m}$ de ancho, ubicada en la base del escarpe de falla. Dentro de esta zona vertical las resistividades son intermedias en valor entre las resistividades altas de la capa superior y las bajas de la capa inferior, que se localizan fuera de la zona de falla.

El perfil de resistividad de la figura 7 es consistente con un origen por falla para el escarpe. Las capas de baja resistividad en ambos lados del escarpe aparecen a una profundidad consistente debajo de la superficie, indicando aproximadamente que un estrato uniforme de espesor uniforme se presenta a ambos lados del escarpe. La consistencia en la profundidad sugiere que el salto vertical en el escarpe de falla es aproximadamente igual al desplazamiento vertical total y de que ha existido relativamente poca erosión del bloque norte levantado.

El origen de la zona de resistividad intermedia encontrada al pie del escarpe de falla no puede ser determinado sin un conocimiento apropiado de terreno. Se especula que las resistividades intermedias provienen de fracturas rellenas con agua y con porosidades más altas en la zona de falla. La presencia de estas fracturas, reemplazando efectivamente matriz con agua, puede actuar para reducir la resistividad de los lahares, mientras incrementaría la resistividad de los sedimentos ricos en arcilla o de los paleosuelos.

El perfil magnético D-D' en la figura 7 se muestra con dos posibles modelos 2,5 D para ver la estructura magnética a través de la falla. Los puntos negros son los datos, las líneas negras son las anomalías magnéticas predichas para el modelo mostrado. Las zonas de gris claro representan material con una susceptibilidad de 0,03 (unidades MKS); la zona gris oscuro tiene una susceptibilidad de 0,04. Los datos magnéticos requieren de una zona superficial de alta susceptibilidad a lo largo del escarpe de falla. Los datos son compatibles con el modelo de una capa desplazada de susceptibilidad aumentada (paleolahares?) mostrada en el perfil del medio y también con el modelo de una zona entre unos 10 a $20 \mathrm{~m}$ de profundidad de alta susceptibilidad subyaciendo el escarpe (aumento de meteorización dentro de la zona de falla?), el cual se muestra en el perfil inferior.

\section{Interpretación de la falla Aguacaliente en los valles de Coris y El Guarco}

En la figura 1 se muestran las trazas de la falla Aguacaliente en los valles de Coris y de El Guarco, luego de integrar la información morfotectónica, geológica, de trincheras, hidrogeológica y geofísica. Una traza de falla es sugerida a lo largo del sector norte del valle de Coris, donde el frente de montaña de los cerros de la Carpintera con el valle de Coris está facetado y es muy lineal. Esta traza parece ser corroborada por el lineamiento magnético que se presenta del lado norte del valle, donde la diferencia entre las ubicaciones entre la traza de falla y el lineamiento magnético se puede explicar por la profundidad que tiene la capa magnética y el buzamiento de la falla (Figs. 2 y 4). La evidencia geomórfica y la interpretación magnética sugieren que es una falla con una componente normal. Esta interpretación es similar a la propuesta por Woodward \& Clyde (1993), quienes determinan una falla en este lado del valle y la consideran sospechosa de ser activa. En Woodward \& Clyde (1993) la traza de esta falla sugerida continúa a lo largo del valle superior de la quebrada Barahona, cerca del contacto entre las formaciones Coris y La Cruz (Fig. 3) y al salir de este valle, limita el frente sur de los cerros de La Carpintera. En este estudio, se sugiere que esta falla sigue el borde norte del valle y más al oeste se une con la traza activa de la falla Aguacaliente, que se ubica del lado sur del valle de Coris (ver abajo). Al este, esta falla puede unirse con la serie de lineamientos cortos de rumbo NE, que tienen facetamientos al $\mathrm{E}$ y que sugieren fallas con una componente normal (Fig. 1). Es posible también que el desvío del río Coris identificado con el 


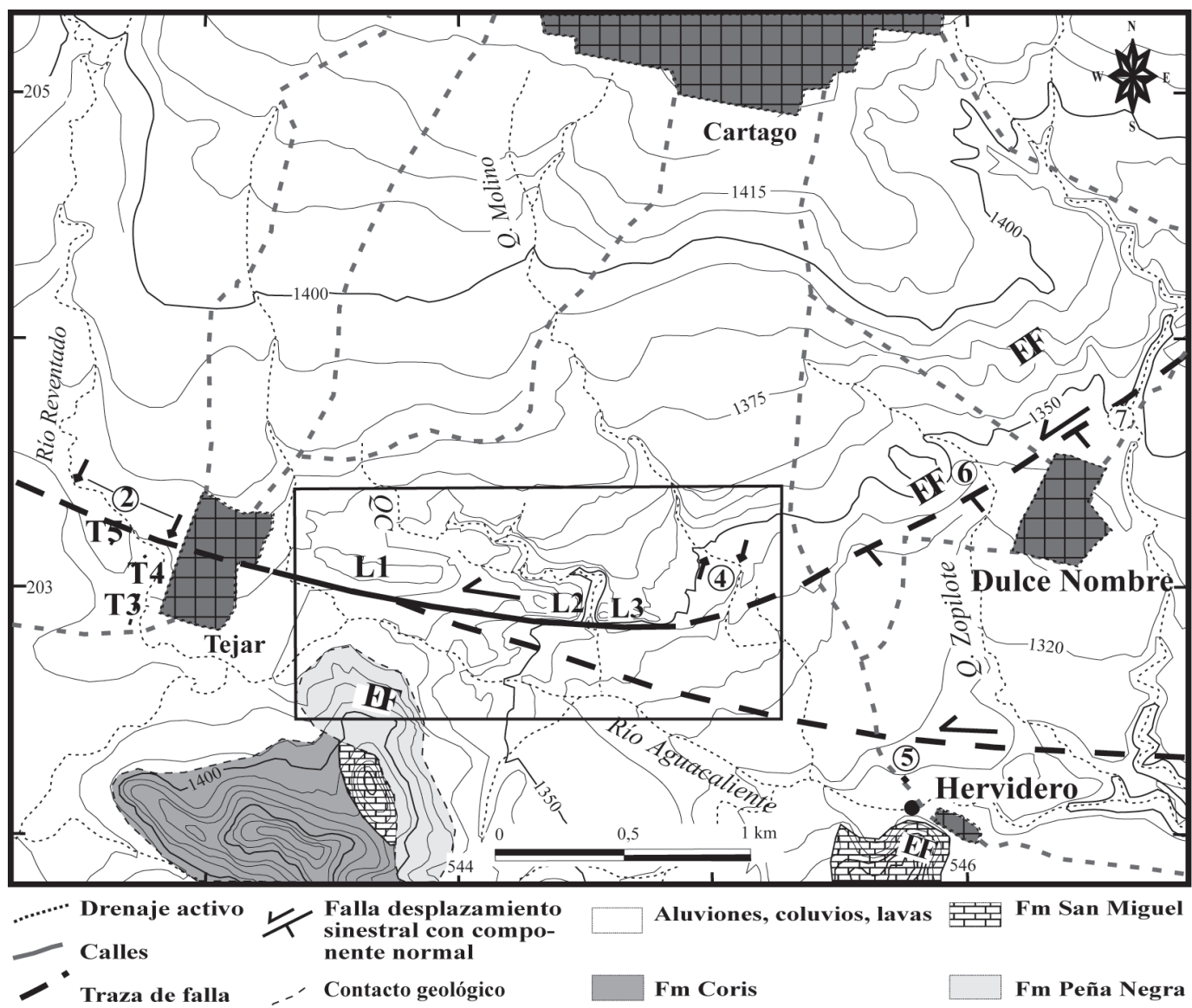

Fig. 5: Mapa geomorfológico y geológico del sur de Cartago. Las curvas topográficas provienen de la hoja Cartago, escala 1: 25000 del IGN. Otras simbologías se muestran en la figura 2. El rectángulo ubica la figura 6A.

número 1 , se relacione con esta traza de falla. $\mathrm{Si}$ este fuera el caso, esta falla sugerida continuaría al E. Al momento actual, no existe evidencia de que esta traza de falla sea activa.

La segunda traza de falla se localiza en la parte sur del valle de Coris (Fig. 2). Esta debe de pasar por la zona de falla ubicada en la trinchera T2. Al lado oeste de T2, esta falla seguiría al oeste del poblado de Coris, cerca del curso de una quebrada afluente de la quebrada Barahona. En esta zona, depósitos cuaternarios estarían en contacto por falla contra las formaciones sedimentarias terciarias, con un aparente desplazamiento izquierdo (Fig. 3). Al este de la trinchera T2, esta traza pasaría del lado norte de los espolones facetados y por las fuentes termales de Bermejo (Figs. 1 y 2). Al este, en la zona de Tejar, la falla debe pasar por el desvío izquierdo del río Reventado y a lo largo de la fila de lomas L1 a L3 (Figs. 1,5 y 6). En este último sector, la falla ha sido determinada con datos de trincheras y los datos magnéticos y especialmente los de resisitividad también concuerdan con la presencia de una falla en esta zona. Esta interpretación es similar a la de Woodward \& Clyde (1993).

Con respecto a la continuación al este de Tejar de la falla Aguacaliente, Woodward \& Clyde (1993) consideran que la falla continúa con un rumbo aproximado WNW (Figs. 1 y 5). Como evidencias de esto se tiene que al noroeste del poblado de Hervidero, en el lado izquierdo de la calle que llega al poblado, se observaron fallas cortando depósitos de tobas posiblemente holocénicas y asociadas con el volcán Turrialba (sitio 

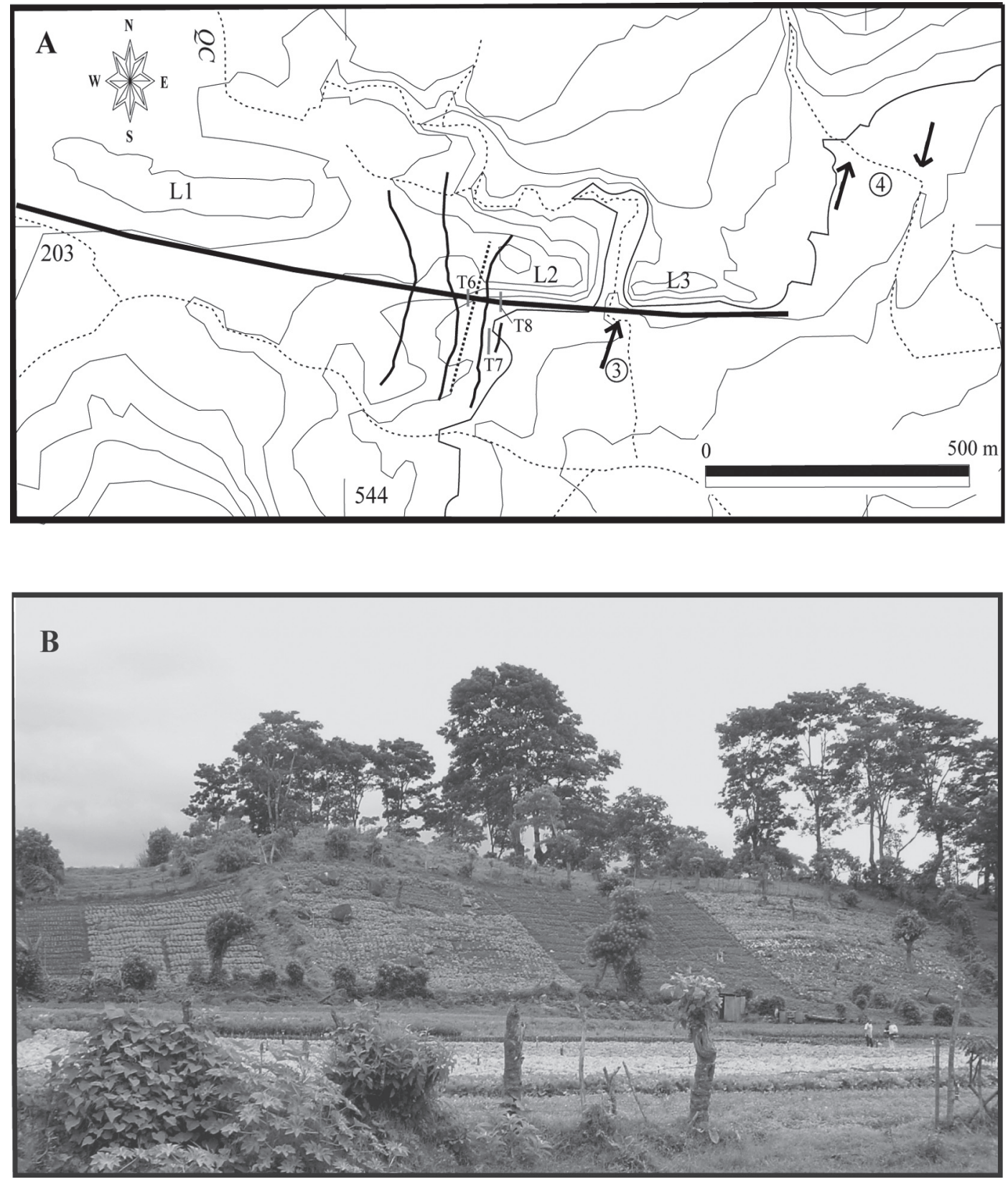

Fig. 6A: Mapa topográfico detallado del lomo de falla del este de Tejar y ubicaciones de trincheras y perfiles geofísicos. Curvas de nivel cada $5 \mathrm{~m}$, tomadas de la hoja Tejar (1: 10 000) del IGN. El perfil de resistividad se muestra como una línea punteada (C-C', Fig. 7) y los cuatro perfiles magnéticos realizados a través del escarpe o al sur de él, se muestran con líneas sólidas. Los cuatro perfiles muestran un gradiente similar de $\mathrm{N}$ a $\mathrm{S}$ con una pronunciada anomalía sobre el escarpe, sugiriendo que el rumbo local tanto del escarpe como de la estructura regional, es E-W. QC es la quebarada Cangrejal.

Fig. 6B: Fotografía de la loma L3 viendo al $\mathrm{N}$ tomada desde el punto 8. 

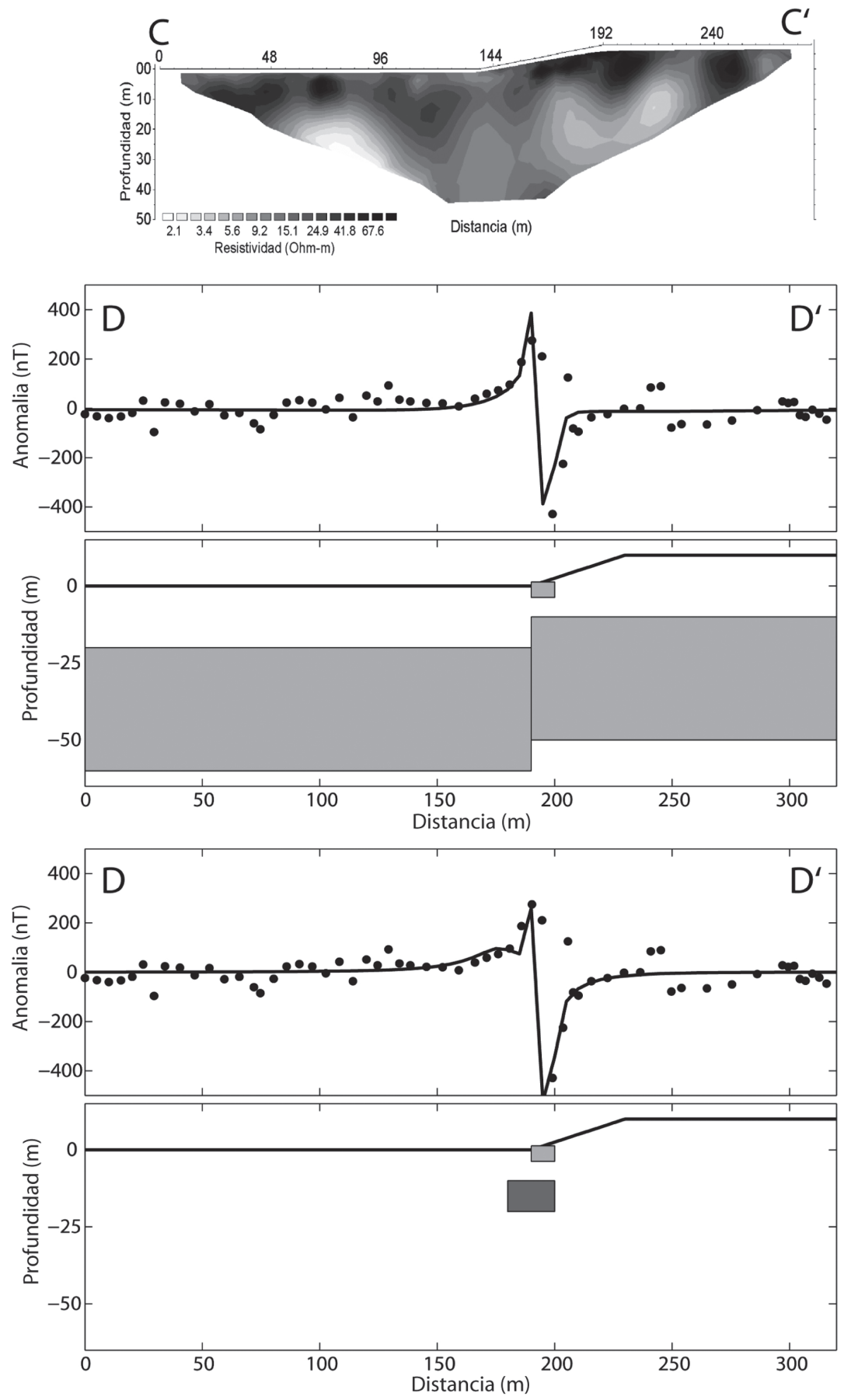

Fig. 7: Inversión de los datos del perfil de resistividad y de los perfiles magnéticos realizados a través del escarpe al E de Tejar (ubicación en Fig. 6). Se muestran dos modelos magnéticos que ajustan adecuadamente los datos del perfil magnético DD'. 
5, Fig. 5). Además, ligeramente al sur del punto anterior se ubica la fuente termal de Hervidero. Más al este y fuera de la zona de estudio se pueden encontrar otros aspectos geomórficos que sugieren esta traza.

Una segunda posibilidad es que la falla continúe con un rumbo noreste al pie de posibles escarpes de falla facetados al este, que se localizan al oeste y al norte de Dulce Nombre (identificados con EF, Fig. 5). En estos sectores, depósitos de lahares se observan aflorando en una zona levantada, lo cual es anómalo considerando que estas unidades se ubicarían en la parte distal del abanico depositado por ríos como el Reventado, cuyo ápice se ubica del lado norte de la ciudad de Cartago. En un afloramiento ubicado en un nuevo residencial en esta zona se encontraron una zona de fracturas y fallas subverticales cortando los lahares (sitio 6, Fig. 5). Una posible evidencia adicional de esta traza la proporcionó el geólogo Álvaro Aguilar (com. pers., 2006), quien nos dio a conocer la existencia de una manifestación termal dentro de la localidad de Dulce Nombre (círculo relleno, Fig. 5). Más al noreste, en la margen derecha del río Toyogres, se encontraron dos secuencias de depósitos de terraza aluvial basculados separados por una discordancia angular. La secuencia inferior tiene un buzamiento aparente de $40^{\circ}$ al SE y los aluviones que sobreyacen tienen un buzamiento aparente de $25^{\circ}$ al SE (sitio 7, Fig. 5). Se interpreta que el basculamiento es originado por el movimiento en una falla cercana con componente normal. Por lo tanto, con los datos anteriores, es posible también sugerir una traza con rumbo noreste a partir de la fila de presión del este de Tejar.

\section{DISCUSIÓN Y CONCLUSIONES}

Empleando datos geológicos, geomorfológicos y geofísicos se han definido las trazas de la falla Aguacaliente en el sector comprendido entre el valle de Coris y el sur de la ciudad de Cartago. Una traza con probable componente normal (bloque del lado sur se hunde relativamente), lo cual es sugerido por la geomorfología y los datos magnéticos, se localiza del lado norte del valle de
Coris en el límite con los cerros de la Carpintera. Más al este, esta traza parece terminar en una serie de fallas de rumbo NE, con componente normal, que están facetadas al este y que por lo tanto sugieren que el lado este se hunde. El conjunto de las anteriores trazas corresponde bastante con las sugeridas originalmente en Woodward \& Clyde (1993). El grado de actividad de estas trazas se desconoce, aunque los datos geomórficos sugieren que pueden estar inactivas.

Según datos geomórficos, de trincheras y geofísicos, la traza principal de la falla Aguacaliente, de un rumbo WNW, se localiza sobre el borde sur de los valles de Coris y de El Guarco (Fig. 1). La actividad reciente de esta traza de falla se concluye de los estudios de trincheras realizados cerca de Coris y de Tejar (Woodward \& Clyde, 1993; Montero et al., 1991) y es sugerida por los estudios de intensidades del terremoto del 4 de mayo de 1910 (Montero \& Miyamura, 1981) y por la actividad sísmica en forma de enjambres ocurrida en los últimos años (Fernández \& Montero, 2002).

De acuerdo con los desvíos dominantemente izquierdos presentes en ríos y quebradas y la interpretación del origen de las fuentes termales de Bermejo, el desplazamiento predominante de la traza activa de la falla es siniestral. Esto concuerda con los datos aportados por Montero et al. (en prensa) para el sector de la falla al sur de San José.

Los datos disponibles permiten determinar con precisión la traza de la falla activa en sectores restringidos de ambos valles, mientras que en otros solo es inferida con base en información geomórfica. La existencia de un relleno cuaternario poco consolidado en ambos valles, en conjunto con una posible baja tasa de actividad en la falla y el tipo de desplazamiento de rumbo que tiene la falla, son factores que explican que la traza de falla tenga prominencia moderada a débil.

La falla tiene una zona de deformación ancha tanto en el valle de Coris como en el de El Guarco. Así, en la trinchera T2 la zona de falla es de unos $40 \mathrm{~m}$ de ancho. Más al este, las fuentes termales de Bermejo se extienden en una zona que tiene entre 120 y $200 \mathrm{~m}$ de ancho. Estas se interpretan asociadas con fallas o fracturas de tensión, lo cual sugiere que se localizan 
dentro de zona ancha de falla sometida a una cupla sinestral. Igualmente, en las trincheras T6 y $\mathrm{T} 8$ se pudo definir una zona ancha de falla, que incluye no solo la zona de la loma L2 sino un sector al sur de esta (Montero et al., 1991).

La existencia de una zona de falla ancha concuerda con la geología de la zona por donde cruza la falla. Así, en el valle de Coris existe una zona de relleno cuaternario con depósitos aluviales, lacustres y de pantano, los cuales tienen en general poca compactación especialmente cerca de la superficie. Una situación similar se encuentra en el sector sur del valle de El Guarco. El único sitio en esta zona donde la falla tiene una expresión geomórfica más prominente es en la fila de lomas L1 a L3 que se ubica al este de Tejar, que es donde la falla corta depósitos de lahares, los cuales tienen una mayor consistencia y dureza. La fila de lomas anterior fue relacionada por Montero et al. (1991) con una transpresión local a lo largo de la falla Agua Caliente, lo cual origina un sistema de fallas inversas dispuestas en una flor positiva. Efectivamente, esta fila se interpreta como un lomo de falla, asociado con un cambio en el rumbo de la falla, debido a una transpresión local.

Una falla de desplazamiento de rumbo al propagarse a través de depósitos poco consolidados puede generar una zona de deformación ancha. Por ejemplo, Tschalenko (1970) encontró en ciertos sectores de la ruptura asociada con el terremoto de Dasht-e-Bayas, ocurrido en Irán en 1968, zonas de fracturas Riedel distribuidas en una zona de un ancho de más de $100 \mathrm{~m}$. Un caso similar se observó en ciertos sectores de la ruptura de la falla Emerson, durante el terremoto de Landers en 1992 (Yeats et al., 1997). En ambos casos, las zonas de ruptura ancha se propagaron a través de depósitos recientes.

La relación tectónica entre las trazas de falla Aguacaliente observadas en el valle de Coris, sugieren que estas forman el ápice de una estructura transtensional (Fig. 1). La falla del lado norte del valle tiene características de tener una componente normal. Asimismo, las fallas de rumbo $\mathrm{NE}$, que limitan los cerros de La Carpintera por su lado oriental, en la transición hacia el valle del Guarco sugieren una componente normal donde el lado E se hunde. Lo anterior podría implicar que los valles de Coris y de El Guarco corresponden con una depresión tipo "pull-apart", sugerencia que merece ser investigada en el futuro. $\mathrm{Si}$ ese fuera el caso, las trazas de falla del norte de Coris y las del lado de oriental de los cerros de La Carpintera estarían actualmente inactivas, como resultado de la evolución del "pull-apart". Esta última estructura hoy día sería cortada por una falla transversal (definida como "cross-basin fault" por McClay \& Dooley, 1995) como es la falla Aguacaliente, la que se ubica del lado sur de los valles de Coris y de El Guarco, pero que al oriente de Tejar cortaría a través del valle. Esta falla de movimiento predominante sinestral, sería la falla donde se concentra actualmente el desplazamiento.

\section{AGRADECIMIENTOS}

Se agradece al Geól. Lepolt Linkimer por la recopilación geológica realizada en la zona de estudio. Asimismo, las conversaciones con el Geól. José William Pérez con el aporte de información sobre la hidrogeología del valle de Coris fueron muy valiosas. Al Geól. Álvaro Aguilar se le agradece información diversa sobre la geología y neotectónica al sur de Cartago. Un agradecimiento a los funcionarios Marvin Aguilar y Carlos Romero de SENARA, por proporcionar información hidrogeológica de la zona de estudio. Especial agradecimiento a los señores Ruperto Molina, Rodrigo Pereira y Francisco Muñoz por permitir el acceso a propiedades donde se realizaron los estudios geofísicos y aportar información de la zona.

\section{REFERENCIAS}

DENYER, P. \& ARIAS, O., 1991: Estratigrafía de la región central de Costa Rica. - Rev. Geol. Amér. Central, 12: 1-59.

DENYER, P. \& MONTERO, W., 1988: Mapa geoestructural y de sismos del Valle Central.Escala 1: 150 000. IGN, San José. 
DÓNDOLI, C. \& TORRES, A., 1954: Estudio geoagronómico de la región oriental de la Meseta Central. - 180 págs. Ministerio de Agricultura e Industria. San José.

FERNÁNDEZ, M. \& MONTERO, W., 2002: Fallamiento y sismicidad del área entre Cartago y San José, valle Central de Costa Rica.- Rev. Geol. Amér. Central, 26: 25-37

LOKE, M. H. \& BARKER, R. D., 1996: Rapid least-squares inversion of apparent resistivity pseudo-sections using quasi-Newton method.- Geophys. Prospect., 48: 181-152.

MCCLAY, K. \& DOOLEY, T., 1995: Analogue models of pull-apart-basins.- Geology, 8: 711-714.

MONTERO, W., 1994: Sismicidad y neotectónica, en: DENYER, P. \& KUSSMAUL, S. (Comp.), 1994: Atlas Geológico Gran Área Metropolitana. - Ed. Tecnológica de Costa Rica: $147-160$.

MONTERO, W., BARAHONA, M., ROJAS, W. \& TAYLOR, M., en prensa: Los sistemas de falla Aguacaliente y Río Azul y relevos compresivos asociados, valle Central de Costa Rica. - Rev. Geol. Amér. Central, 33: 7-27.

MONTERO, W. \& MIYAMURA, S., 1981: Distribución de intensidades y estimación de los parámetros focales de los terremotos de Cartago de 1910, Costa Rica, América Central.- Rev. Inst. Geogr. Nacional, JulioDiciembre: 9-34.
MONTERO, W., OBANDO, L., MORA, R., SALAZAR, L. G. \& LEANDRO G., 1991: Informe Final Proyectos Habitacionales Los Diques y Duarco-Suerre.- 72 págs. Escuela Centroamericana de Geología, Universidad de Costa Rica.

MORALES, L. D., 1975 : Estudio geológico de la esquina suroeste de la hoja Istarú. - 25 págs. Univ. de Costa Rica, San José [Inf. Campaña Geol.].

PÉREZ, J. W., 2000 : Estudio hidrogeológico e hidroquímico preliminar para evaluar la contaminación de aguas superficiales y subterráneas del valle de CorisProvincia de Cartago.- 38 págs + figuras. Servicio Nacional de Aguas Subterráneas, Riego y Avenamiento, Área de Aguas Subterráneas.

TCHALENKO, J., 1970: Similarities between shear zones of different magnitudes. - Geol. Soc. Amer. Bull. 81: 1625-1640.

WOODWARD \& CLYDE, 1993: A preliminary evaluation of earthquake and volcanic hazards significant to the major population centers of the Valle Central, Costa Rica. - 89 págs. Final Report prepared for Ret Corporation.

YEATS, R. S., SIEH, K. \& ALLEN, C. R., 1997: The Geology of Earthquakes.- 568 págs. Oxford University Press, Nueva York. 